\title{
Fatores Associados à Suscetibilidade para o Desenvolvimento de Transtornos Alimentares em Estudantes Internos de um Curso de Medicina
}

\section{Factors Associated to Susceptibility for the Development of Eating Disorders among Medical Interns}

\author{
Mariana de Oliveira Inocente Aidar ${ }^{I}$ (iD \\ Rafaela Borges de Freitas ${ }^{I}$ (D) \\ Gabriela Cunha Fialho Cantarelli Bastos ${ }^{I}(\mathbb{D}$ \\ Aline Alves Brasileiro ${ }^{I} \mathbb{D}$ \\ Antonio Márcio Teodoro Cordeiro Silva ${ }^{I}$ (iD \\ Rogério José de Almeida ${ }^{I}$ (iD
}

\section{PALAVRAS-CHAVE}

- Estudantes de Medicina.

- Transtornos Alimentares.

- Anorexia Nervosa.

- Bulimia Nervosa.
Introdução: O objetivo deste estudo foi analisar os fatores associados à suscetibilidade para o desenvolvimento de transtornos alimentares em estudantes internos de um curso de Medicina. Método: Trata-se de um estudo transversal analítico com abordagem quantitativa, em que se aplicaram dois questionários: um com dados sociodemográficos e outro com o Teste de Atitudes Alimentares (EAT-26), que é um instrumento psicométrico para triar transtornos alimentares. Resultados: Foram incluídos na pesquisa 162 estudantes internos de Medicina. No gênero feminino, identificaram-se maiores escores no EAT-26 na escala da dieta $(D)(p=0,0079)$, que evidencia uma recusa patológica a comidas hipercalóricas e uma excessiva preocupação com a forma física, na escala de bulimia e preocupação com os alimentos $(B)(p=0,0014)$ e no escore geral da EAT-26 $(p=0,0005)$. Maior escore na escala $D$ foi encontrado em estudantes que trabalham e estudam ( $p=0,0278)$ e naqueles com sobrepeso ( $p=0,0297)$. Aqueles que referiam estar seguindo alguma dieta tiveram maiores escores na escala $D(p<0,0001)$, na escala $B(p=0,0300)$ e no escore geral $(p=0,0001)$. Os que afirmaram ter preocupação quanto à quantidade de calorias obtiveram maiores escore na escala $D(p<0,0001)$, na escala $B(p=0,0010)$ e no escore geral $(p<0,0001)$. Os que referiram ter medo de engordar tiveram maiores escores na escala $D$ $(p<0,0001)$, na escala $B(p=0,0001)$ e no escore geral $(p<0,0001)$, e, em contrapartida, aqueles que não tinham medo de engordar obtiveram maior escore na escala controle oral $(C O)(p=0,0149)$, que reflete o autocontrole associado aos alimentos e reconhece influências sociais do meio em que o indivíduo está inserido em relação à ingesta alimentar, assim como aqueles abaixo do peso $(p=0,0042)$. Os ansiosos obtiveram maiores escores na escala $D(p=0,0356)$, na escala $B(p=0,0266)$ e no escore geral $(p=0,0310)$. Conclusões: Ficaram evidenciados maiores escores na escala EAT-26 em estudantes internos de Medicina que são do sexo feminino, que trabalham e estudam e naqueles com sobrepeso. Além disso, seguir alguma dieta, possuir preocupação quanto à quantidade de calorias, ter medo de engordar, ser ansioso, triste e insatisfeito com próprio corpo também foram fatores associados com maiores escores. Todos esses fatores podem ser relacionados com um maior risco de esses estudantes desenvolverem distúrbios alimentares, como a anorexia e a bulimia. 


\section{KEYWORDS}

- Medical Students.

- Eating Disorders.

- Anorexia Nervosa.

- Bulimia Nervosa.

Recebido em 4/5/20

Aceito em 18/6/20

\section{ABSTRACT}

Introduction: To analyze factors associated with susceptibility to the development of eating disorders in medical interns. Methods: This is an analytical cross-sectional study with a quantitative approach. Two questionnaires were applied, one with sociodemographic data and the other with the Eating Attitudes Test (EAT-26), which is a psychometric instrument to screen eating disorders. Results: A total of 162 internal students were included in the research. Higher EAT-26 scores were found in females on the diet scale $(D)(p=0.0079)$, which shows a pathological refusal to hypercaloric foods and an excessive concern with fitness, bulimia scale and concern with food $(B)(p=0.0014)$ and overall EAT-26 score $(p=0.0005)$. Higher $D$-scores were found in working and studying students $(p=0.0278)$ and overweight students $(p=0.0297)$. Those who reported being on some form of diet had higher scores on the $D$ scale $(p<0.0001)$, the $B$ scale $(p=0.0300)$ and the overall score $(p=0.0001)$. Those who said they were concerned about the number of calories had higher scores on the D scale $(p<0.0001)$, $B$ scale $(p=0.0010)$ and overall score $(p<0.0001)$. Those who reported being afraid of getting fat scored higher on the D scale $(p<0.0001), B$ scale $(p=0.0001)$, overall score $(p<0.0001)$, whereas those not afraid of getting fat scored higher on the oral control (CO) scale ( $p=0.0149)$, which reflects self-control associated with food and recognizes social influences of the individual's environment in relation to food intake, as well as those underweight ( $p=0.0042)$. Anxiety patients obtained higher scores on the D scale $(p=0.0356), B$ scale $(p=0.0266)$ and overall score $(p=0.0310)$. Conclusion: Higher scores on the EAT-26 scale were evidenced in medical interns who are female, who work and study, and in overweight patients. Furthermore, following a specific diet, being concerned about the amount of calories consumed, being afraid of getting fat, being anxious, sad and dissatisfied with one's own body were also factors associated with higher scores. All of these factors can be related to an increased risk of these students developing eating disorders, such as anorexia and bulimia.

\footnotetext{
Aceito em $18 / 6 / 20$
}

\section{INTRODUÇÃO}

Os ideais de corpo e saúde instituídos pela sociedade nem sempre foram os mesmos. Novos estereótipos fazem com que o padrão estético atual seja diferente daquele estabelecido no início do século $\mathrm{XX}$. A supervalorização de um corpo magro é vista como sinal de saúde, podendo criar, assim, uma situação de frustração, baixa autoestima e discriminação entre aqueles que não se enquadram nessa regra ${ }^{1-3}$.

Surge, em muitos casos, a preocupação exacerbada com o peso e com a imagem corporal. Essa constante busca por um padrão estético é caracterizada como um dos principais estímulos para comportamentos de risco, os quais podem resultar no desenvolvimento de transtornos alimentares $^{2,4}$.

Os transtornos alimentares são distúrbios psiquiátricos de etiologia multifatorial, caracterizados por consumo, padrões e atitudes alimentares extremamente distorcidos e de preocupação exagerada com o peso e a forma corporal ${ }^{2}$. Eles podem ter várias classificações, sendo as mais prevalentes a anorexia nervosa e a bulimia nervosa ${ }^{2}$.

A anorexia nervosa é desenvolvida, principalmente, em meninas adolescentes (podendo se agravar na vida adulta quando não é corretamente diagnosticada e tratada) e em mulheres jovens. É caracterizada por uma grave restrição da ingestão alimentar, uma busca intensa pela magreza e distorção da imagem corporal. Já a bulimia nervosa caracteriza-se por episódios recorrentes de uma ingestão copiosa de alimentos em um curto período associada a uma sensação de perda de controle (episódios bulímicos), que buscam saciar a fome excessiva e atender aos estados emocionais e às situações de estresse. Esses episódios são acompanhados de métodos compensatórios inadequados para o controle de peso, como: vômitos autoinduzidos, uso de medicamentos, dietas inadequadas e prática de exercícios físicos extenuantes. Diferentemente da anorexia nervosa, na bulimia nervosa não ocorre necessariamente a perda de peso, o que dificulta a deteç̧ão do problema por médicos e familiares ${ }^{2}$.

O diagnóstico de transtorno alimentar deve ser feito por um especialista por meio de uma anamnese dirigida, com exames complementares e com o auxílio de questionários que servem como instrumentos de triagem, como o Teste de Atitudes Alimentares, do inglês Eating Attitudes Test (EAT). Esse rastreamento deve ser feito de forma precoce para prevenir e reduzir os danos somáticos e psíquicos ${ }^{3}$.

Os transtornos alimentares, que podem estar relacionados a outros problemas de saúde mental, como a depressão e ansiedade, são altamente prevalentes nos câmpus universitários; se não forem tratados, poderão gerar consequências físicas, psicológicas, sociais e acadêmicas ${ }^{5}$. Com relação aos universitários, os estudantes de Medicina fazem parte de um grupo de risco para o desenvolvimento de transtornos alimentares. Como causas da significativa taxa de prevalência de distúrbios alimentares entre estudantes de Medicina, é possível constatar um alto nível de estresse devido a uma alta carga horária, atividades curriculares, extracurriculares, autocobrança, distorção da imagem corporal, excesso de peso ou estado nutricional inadequado (sobrepeso ou obesidade), supervalorização do peso e práticas inadequadas de controle do peso ao longo dos anos. Esses fatores de risco refletem diretamente e de forma significativa no aparecimento dos sintomas que podem iniciar na infância e se pronunciarem na fase de vida universitária ${ }^{5}$.

Ademais, a presença de transtornos alimentares nesse grupo está correlacionada com alguns problemas de saúde mental bastante frequentes nos estudantes de Medicina, como a depressão e a ansiedade, bem como com os mecanismos compensatórios do estresse, como a drunkorexia, o

REVISTA BRASILEIRA DE EDUCAÇÃO MÉDICA

2 44 (3) : e097; 2020 
uso de drogas (lícitas e ilícitas) e a vigorexia ${ }^{6}$.

Identificar os fatores associados aos transtornos alimentares em estudantes de Medicina pode contribuir para a identificação das características em comum dos indivíduos que apresentam esses transtornos. Nesse sentido, o presente estudo teve por objetivo analisar os fatores associados à suscetibilidade para o desenvolvimento de transtornos alimentares em estudantes internos de um curso de Medicina.

\section{MÉTODOS}

Trata-se de um estudo transversal analítico com abordagem quantitativa. Esse é um método de pesquisa no qual a exposição e a condição de saúde do participante são determinadas simultaneamente. A característica fundamental desse tipo de estudo é a impossibilidade de saber se a exposição antecede ou é consequência da doença/condição relacionada à saúde ${ }^{7}$.

A pesquisa foi realizada por meio de questionários aplicados em estudantes que estavam cursando o período do curso de Medicina denominado de internato, ou seja, todos aqueles que estavam no nono, décimo, décimo primeiro e décimo segundo períodos do curso de Medicina de uma universidade localizada na cidade de Goiânia, Goiás.

Como critérios de inclusão, pesquisaram-se homens e mulheres, maiores de 18 anos, que estavam matriculados do nono ao décimo segundo período do curso de Medicina, no período da coleta de dados, que ocorreu entre setembro e outubro de 2018, e que estavam frequentando regularmente as aulas. Excluíram-se aqueles que não responderam a todas as questões dos questionários e que se encontravam com a matrícula trancada.

Foram utilizados nesta pesquisa dois instrumentos. Adotou-se um questionário sociodemográfico que contemplou as seguintes variáveis: idade, sexo, etnia, estado civil, religião, altura autorreferida e peso autorreferido, prática ou não de atividade física, se o aluno mora sozinho ou não, se a família mora em outra cidade, prática ou não de dieta, se existe preocupação com a quantidade calórica dos alimentos, se existe uma preocupação em não engordar, entre outras.

Adotou-se também o EAT-26 que é um instrumento psicométrico utilizado para triar transtornos alimentares com o intuito de medir sintomas de forma eficaz, de modo a favorecer o diagnóstico e o tratamento precoces e impedir a evolução da doença. Desenvolvido em $1979^{8}$, o EAT já foi adaptado e validado no Brasil ${ }^{9}$.

A versão original do EAT (EAT-40) era composta por 40 itens de múltipla escolha. $\mathrm{O}$ resultado do teste era o somatório dos valores atribuídos às questões, com um ponto de corte de 30 pontos, o que era um indicador positivo para uma possível existência de distúrbio alimentar. Posteriormente, foi proposta a versão abreviada (EAT- 26) com 26 itens, na qual o ponto de corte passou a ser de 21 pontos $^{10}$.

As 26 questões são divididas em três fatores:

- Escala da dieta (D): itens $\mathrm{n}^{\circ} 1,6,7,10,11,12,14,16,17,22$ 23, 24, 25 que evidenciam uma recusa patológica a comidas hipercalóricas e uma excessiva preocupação com a forma física.

- Escala de bulimia e preocupação com os alimentos (B): itens $\mathrm{n}^{\circ}$ $3,4,9,18,21,26$ que são relacionados aos episódios de ingesta compulsória dos alimentos, seguidos de vômitos e outras medidas para evitar o ganho de peso.
- Escala do controle oral (CO): itens no 2, 5, 8, 13, 15, 19, 20 que refletem o autocontrole associado aos alimentos e reconhecem influências sociais, em relação à ingesta alimentar, do meio em que o indivíduo está inserido?.

Cada questão, dividida em três escalas do tipo Likert, possui seis opções de resposta, com um escore de 0 a 3, dependendo da escolha (sempre $=3$ pontos, muitas vezes $=2$ pontos, às vezes $=1$ ponto, poucas vezes/ quase nunca/ nunca $=0$ pontos), e a única questão que apresenta pontos em ordem invertida é a $25^{9}$.

Após a coleta de dados, foi confeccionado um banco de dados no software Excel. Posteriormente, utilizou-se o software BioEstat 3.0 para realizar estatística descritiva com o cálculo medidas de tendência central para as variáveis contínuas, como média e desvio padrão, bem como o cálculo das frequências absoluta e relativa percentual para as variáveis discretas.

Em seguida, aplicou-se teste de normalidade (D'Agostino-Pearson) para distinguir as distribuições paramétricas e não paramétricas, com o intuito de comparação dos resultados do questionário estratificado pelas variáveis sociodemográficas. Para as distribuições paramétricas, utilizaram-se os testes $\mathrm{t}$ de Student e ANOVA, e, para as distribuições não paramétricas, os testes Mann-Whitney e Kruskal-Wallis. Para todos os testes comparativos, foi assumido p-valor inferior ou igual a 0,05 como significativo.

De acordo com a Resolução no 466/2012, a pesquisa foi registrada na Plataforma Brasil do Ministério da Saúde, com o Certificado de Apresentação para Apreciação Ética (CAAE) no 90495718.5.0000.0037, e aprovada pelo Comitê de Ética em Pesquisa da Pontifícia Universidade Católica de Goiás (PUC Goiás) com o Parecer n 2.723.391.

\section{RESULTADOS}

Foram analisados 162 questionários de estudantes internos de um curso de Medicina, os quais evidenciaram que, em relação aos aspectos sociodemográficos, a maioria era do sexo feminino (55,6\%), com até 24 anos $(63,6 \%)$, solteira $(54,9 \%)$, adepta de uma religião $(75,3 \%)$ e morava com familiares $(80,9 \%)$ (Tabela 1$)$.

Em relação aos aspectos pessoais e clínicos dos estudantes pesquisados, observou-se que $70,4 \%$ apresentavam peso normal, enquanto $21,6 \%$ eram considerados com sobrepeso. Ademais, $77,8 \%$ relataram que não seguiam alguma dieta, apesar de 73,5\% afirmarem que tinham medo de engordar. Quanto à prática de atividade física, $41,4 \%$ declararam que realizavam frequentemente. Dos alunos pesquisados, $87,7 \%$ não tinham doenças crônicas. Além disso, 90,7\% não se consideravam tristes e 52,5\% estavam satisfeitos com o próprio corpo (Tabela 2).

Com referência ao EAT, um total de 37 (22,8\%) da amostra apresentou escore superior ou igual a 21, indicando transtorno alimentar (Tabela 3). $\mathrm{Na}$ comparação do escore geral do EAT e dos fatores da escala com as variáveis sociodemográficas, identificaram-se com significância estatística maiores escores do sexo feminino em relação ao masculino na escala $D$ $(p=0,0079)$, na escala $B(p=0,0014)$ e no escore geral do EAT-26 $(p=$ $0,0005)$. Maior escore na escala $D$ também foi encontrado em estudantes que afirmaram que trabalhavam e estudavam $(\mathrm{p}=0,0278)$ (Tabela 4$)$.

No que se refere à comparação dos aspectos pessoais e clínicos com os escores do EAT-26 e de seus fatores, identificou-se um maior escore na escala $D$ nos estudantes com sobrepeso $(p=0,0297)$ e, na escala $C O$, naqueles abaixo do peso $(p=0,0042)$. Aqueles que referiam estar seguindo

REVISTA BRASILEIRA DE EDUCAÇÃo MÉDICA

3 44 (3) : e097; 2020 
alguma dieta tiveram maiores escores na escala $\mathrm{D}(\mathrm{p}<0,0001)$, na escala B $(p=0,0300)$ e no escore geral $(p=0,0001)$ (Tabela 5$)$.

Os estudantes que afirmaram ter preocupação quanto à quantidade de calorias obtiveram maiores escores na escala D ( $p<0,0001)$, na escala B (p $=0,0010)$ e no escore geral $(p<0,0001)$. Semelhante resultado também foi encontrado naqueles que referiram ter medo de engordar, já que tiveram maiores escores na escala D $(p<0,0001)$, na escala $B(p=0,0001)$ e no escore geral ( $\mathrm{p}<0,0001)$, e, em contrapartida, aqueles que não tinham medo de engordar obtiveram maior escore na escala CO $(p=0,0149)$ (Tabela 5).

\begin{tabular}{|c|c|c|}
\hline \multicolumn{3}{|c|}{$\begin{array}{c}\text { Tabela } 1 \\
\text { Caracterização dos aspectos sociodemográficos dos } 162 \text { estudantes } \\
\text { internos de um curso de Medicina de Goiânia, Goiás, Brasil, } 2018\end{array}$} \\
\hline Variáveis & $\mathbf{N}$ & $\mathrm{f}(\%)$ \\
\hline \multicolumn{3}{|l|}{ Sexo } \\
\hline Masculino & 72 & 44,4 \\
\hline Feminino & 90 & 55,6 \\
\hline \multicolumn{3}{|l|}{ Idade (anos) } \\
\hline Até 24 & 103 & 63,6 \\
\hline Acima de 24 & 59 & 36,4 \\
\hline Média (DP) & 24,5 & 2,7 \\
\hline Mín.-máx. & 21 & 39 \\
\hline \multicolumn{3}{|l|}{ Procedência } \\
\hline Goiânia & 103 & 63,6 \\
\hline Interior de Goiás & 41 & 25,3 \\
\hline Outro estado & 18 & 11,1 \\
\hline \multicolumn{3}{|l|}{ Cursando } \\
\hline Módulo 9 & 55 & 34,0 \\
\hline Módulo 10 & 35 & 21,6 \\
\hline Módulo 11 & 39 & 24,1 \\
\hline Módulo 12 & 33 & 20,4 \\
\hline \multicolumn{3}{|l|}{ Estado civil } \\
\hline Solteiro & 89 & 54,9 \\
\hline Solteiro (namorando) & 64 & 39,5 \\
\hline Casado & 9 & 5,6 \\
\hline \multicolumn{3}{|l|}{ Trabalha e estuda } \\
\hline Sim & 43 & 26,5 \\
\hline Não & 119 & 73,5 \\
\hline \multicolumn{3}{|l|}{ Religião } \\
\hline Sim & 122 & 75,3 \\
\hline Não & 40 & 24,7 \\
\hline \multicolumn{3}{|l|}{ Com quem mora } \\
\hline Sozinho & 23 & 14,2 \\
\hline Familiares & 131 & 80,9 \\
\hline Amigos & 1 & 0,6 \\
\hline Parentes & 7 & 4,3 \\
\hline
\end{tabular}

Fonte: Elaborada pelos autores.

\begin{tabular}{|c|c|c|}
\hline \multicolumn{3}{|c|}{$\begin{array}{c}\text { Tabela } 2 \\
\text { Caracterização dos aspectos pessoais e clínicos dos } 162 \text { estudantes } \\
\text { internos de um curso de Medicina de Goiânia, Goiás, Brasil, } 2018\end{array}$} \\
\hline Variáveis & $\mathrm{n}$ & $f(\%)$ \\
\hline \multicolumn{3}{|l|}{ IMC (kg/m2) } \\
\hline Abaixo do peso $(<18,5)$ & 4 & 2,5 \\
\hline Eutrofia $(18,5-24,9)$ & 114 & 70,4 \\
\hline Sobrepeso $(25,0-29,9)$ & 35 & 21,6 \\
\hline Obesidade $(\geq 30,0)$ & 6 & 3,7 \\
\hline \multicolumn{3}{|l|}{ Segue alguma dieta } \\
\hline Sim & 36 & 22,2 \\
\hline Não & 126 & 77,8 \\
\hline \multicolumn{3}{|l|}{ Preocupação quanto à caloria } \\
\hline Sim & 94 & 58,0 \\
\hline Não & 68 & 42,0 \\
\hline \multicolumn{3}{|l|}{ Medo de engordar } \\
\hline Sim & 119 & 73,5 \\
\hline Não & 43 & 26,5 \\
\hline \multicolumn{3}{|l|}{ Frequenta bares e festas } \\
\hline Frequentemente & 52 & 32,1 \\
\hline Às vezes & 70 & 43,2 \\
\hline Raramente & 40 & 24,7 \\
\hline \multicolumn{3}{|l|}{ Realiza atividade física } \\
\hline Frequentemente & 67 & 41,4 \\
\hline Às vezes & 40 & 24,7 \\
\hline Raramente & 55 & 34,0 \\
\hline \multicolumn{3}{|l|}{ Tem doença crônica } \\
\hline Sim & 20 & 12,3 \\
\hline Não & 142 & 87,7 \\
\hline \multicolumn{3}{|l|}{ Considera-se ansioso } \\
\hline Sim & 107 & 66,0 \\
\hline Não & 55 & 34,0 \\
\hline \multicolumn{3}{|l|}{ Considera-se triste } \\
\hline Sim & 15 & 9,3 \\
\hline Não & 147 & 90,7 \\
\hline \multicolumn{3}{|l|}{ Satisfação com o corpo } \\
\hline Satisfeito & 85 & 52,5 \\
\hline Pouco satisfeito & 62 & 38,3 \\
\hline Insatisfeito & 15 & 9,3 \\
\hline \multicolumn{3}{|l|}{ Ingestão de álcool } \\
\hline Sim & 108 & 66,7 \\
\hline Não & 54 & 33,3 \\
\hline \multicolumn{3}{|l|}{ Fumante } \\
\hline Sim & 11 & 6,8 \\
\hline Não & 151 & 93,2 \\
\hline
\end{tabular}

Fonte: Elaborada pelos autores. 
Tabela 3

Análise dos escores do EAT dos 162 estudantes internos de um curso de Medicina de Goiânia, Goiás, Brasil, 2018

\section{Variáveis}

$\mathbf{N}$

f $(\%)$

Escore do EAT-26

$\begin{array}{lcc}<21 & 125 & 77,2 \\ \geq 21 & 37 & 22,8\end{array}$

Aqueles que se disseram ser ansiosos tiveram maiores escores na escala $D(p=0,0356)$, na escala $B(p=0,0266)$ e no escore geral $(\mathrm{p}=$ $0,0310)$. Os que se consideravam tristes foram identificados com média mais alta no escore geral $(\mathrm{p}=0,0290)$. Os estudantes de Medicina que afirmaram estar insatisfeitos com o próprio corpo tiveram maiores escores na escala D ( $\mathrm{p}<0,0001)$, na escala $\mathrm{B}(\mathrm{p}<0,0001)$ e no escore geral ( $\mathrm{p}$ $=0,0005)$. Aqueles que não ingerem bebida alcoólica obtiveram maior escore na escala CO ( $\mathrm{p}=0,0407)$ (Tabela 5).

Fonte: Elaborada pelos autores.

\section{Tabela 4}

Comparação dos escores do EAT e de seus fatores com os aspectos sociodemográficos dos 162 estudantes internos de um curso de Medicina de Goiânia, Goiás, Brasil, 2018

\begin{tabular}{|c|c|c|c|c|c|c|c|c|c|c|c|c|}
\hline \multirow{2}{*}{ Variável } & \multicolumn{2}{|c|}{ Escala D } & \multirow{2}{*}{ p-valor } & \multicolumn{2}{|c|}{ Escala B } & \multirow{2}{*}{ p-valor } & \multicolumn{2}{|c|}{ Escala CO } & \multirow{2}{*}{ p-valor } & \multicolumn{2}{|c|}{ Escore geral } & \multirow{2}{*}{ p-valor } \\
\hline & Média & DP & & Média & DP & & Média & DP & & Média & DP & \\
\hline \multicolumn{13}{|l|}{ Sexo } \\
\hline Masculino & 6,2 & 5,3 & & 2,1 & 1,9 & & 3,3 & 2,6 & & 11,5 & 6,7 & \\
\hline Feminino & 9,5 & 7,6 & 0,0079 & 3,4 & 2,6 & 0,0014 & 4,0 & 3,4 & 0,2560 & 16,9 & 10,2 & 0,0005 \\
\hline \multicolumn{13}{|l|}{ Idade (anos) } \\
\hline Até 24 & 7,9 & 6,9 & & 2,7 & 2,2 & & 3,7 & 3,0 & & 14,3 & 9,2 & \\
\hline Acima de 24 & 8,4 & 6,8 & 0,5413 & 2,9 & 2,7 & 0,8701 & 3,6 & 3,2 & 0,8577 & 14,9 & 9,3 & 0,5084 \\
\hline \multicolumn{13}{|l|}{ Procedência } \\
\hline Goiânia & 8,2 & 6,8 & & 2,8 & 2,2 & & 3,5 & 3,2 & & 14,5 & 9,2 & \\
\hline Interior de Goiás & 8,1 & 7,4 & & 3,1 & 2,7 & & 4,4 & 3,0 & & 15,6 & 9,8 & \\
\hline Outro estado & 6,9 & 6,2 & 0,7575 & 2,2 & 2,4 & 0,3951 & 2,8 & 1,7 & 0,0832 & 11,9 & 7,7 & 0,3933 \\
\hline \multicolumn{13}{|l|}{ Cursando } \\
\hline Módulo 9 & 7,6 & 6,8 & & 2,6 & 2,3 & & 3,6 & 3,0 & & 13,8 & 8,8 & \\
\hline Módulo 10 & 9,5 & 6,8 & & 3,0 & 2,3 & & 4,0 & 3,5 & & 16,5 & 9,4 & \\
\hline Módulo 11 & 8,0 & 7,7 & & 3,0 & 2,8 & & 3,7 & 3,1 & & 14,6 & 10,9 & \\
\hline Módulo 12 & 7,4 & 6,1 & 0,4402 & 2,6 & 2,2 & 0,8096 & 3,5 & 2,9 & 0,9809 & 13,6 & 7,4 & 0,5093 \\
\hline \multicolumn{13}{|l|}{ Estado civil } \\
\hline Solteiro & 8,0 & 6,5 & & 2,7 & 2,2 & & 3,5 & 2,9 & & 14,2 & 9,0 & \\
\hline Solteiro (namorando) & 8,3 & 7,3 & & 3,0 & 2,6 & & 3,7 & 3,0 & & 15,0 & 9,1 & \\
\hline Casado & 6,8 & 7,8 & 0,6408 & 2,6 & 2,9 & 0,7735 & 5,4 & 4,9 & 0,5336 & 14,8 & 12,6 & 0,7728 \\
\hline \multicolumn{13}{|l|}{ Trabalha e estuda } \\
\hline Sim & 9,7 & 7,0 & & 3,0 & 2,2 & & 3,2 & 2,9 & & 15,8 & 9,7 & \\
\hline Não & 7,5 & 6,7 & 0,0278 & 2,7 & 2,5 & 0,3478 & 3,9 & 3,1 & 0,1442 & 14,1 & 9,0 & 0,3344 \\
\hline \multicolumn{13}{|l|}{ Religião } \\
\hline Sim & 8,4 & 7,2 & & 2,8 & 2,5 & & 3,7 & 3,2 & & 14,9 & 9,7 & \\
\hline Não & 7,0 & 5,5 & 0,5786 & 2,9 & 2,2 & 0,5733 & 3,6 & 2,7 & 0,7092 & 13,5 & 7,7 & 0,7753 \\
\hline \multicolumn{13}{|l|}{ Envolvimento religioso } \\
\hline Forte & 8,8 & 8,2 & & 2,9 & 2,7 & & 4,4 & 3,8 & & 16,1 & 11,4 & \\
\hline Fraco & 8,2 & 6,7 & & 2,7 & 2,3 & & 3,3 & 2,5 & & 14,1 & 8,3 & \\
\hline Nenhum & 7,3 & 4,8 & 0,9982 & 2,5 & 1,8 & 0,9899 & 2,6 & 2,3 & 0,2439 & 12,5 & 6,4 & 0,7851 \\
\hline \multicolumn{13}{|l|}{ Mora } \\
\hline Sozinho & 10,3 & 8,8 & & 3,1 & 3,2 & & 3,8 & 3,2 & & 17,2 & 12,0 & \\
\hline Familiares & 7,9 & 6,5 & & 2,8 & 2,3 & & 3,7 & 3,1 & & 14,4 & 8,7 & \\
\hline Parentes & 3,6 & 3,5 & 0,1118 & 1,9 & 1,8 & 0,6161 & 2,7 & 2,9 & 0,6305 & 8,1 & 4,8 & 0,1108 \\
\hline
\end{tabular}

Fonte: Elaborada pelos autores. 


\section{Tabela 5}

Comparação dos escores do EAT e de seus fatores com os aspectos pessoais e clínicos dos 162 estudantes internos de um curso de Medicina de Goiânia, Goiás, Brasil, 2018

\begin{tabular}{|c|c|c|c|c|c|c|c|c|c|c|c|c|}
\hline \multirow{2}{*}{ Variável } & \multicolumn{2}{|c|}{ Escala D } & \multirow{2}{*}{ p-valor } & \multicolumn{2}{|c|}{ Escala B } & \multirow{2}{*}{ p-valor } & \multicolumn{2}{|c|}{ Escala CO } & \multirow{2}{*}{ p-valor } & \multicolumn{2}{|c|}{ Escore geral } & \multirow{2}{*}{ p-valor } \\
\hline & Média & DP & & Média & DP & & Média & DP & & Média & DP & \\
\hline \multicolumn{13}{|l|}{ IMC $\left(\mathrm{kg} / \mathrm{m}^{2}\right)$} \\
\hline Abaixo do peso $(<18,5)$ & 0,8 & 1,0 & & 1,0 & 2,0 & & 8,5 & 5,4 & & 10,3 & 3,4 & \\
\hline Eutrofia $(18,5-24,9)$ & 8,1 & 7,2 & & 2,9 & 2,4 & & 4,0 & 3,1 & & 15,0 & 9,7 & \\
\hline Sobrepeso $(25,0-29,9)$ & 8,9 & 6,0 & & 2,6 & 2,3 & & 2,7 & 2,3 & & 14,1 & 8,5 & \\
\hline Obesidade $(\geq 30)$ & 6,7 & 4,5 & 0,0297 & 2,0 & 2,6 & 0,2058 & 1,5 & 1,0 & 0,0042 & 10,2 & 5,9 & 0,5970 \\
\hline \multicolumn{13}{|l|}{ Mora } \\
\hline Sozinho & 10,3 & 8,8 & & 3,1 & 3,2 & & 3,8 & 3,2 & & 17,2 & 12,0 & \\
\hline Familiares & 7,9 & 6,5 & & 2,8 & 2,3 & & 3,7 & 3,1 & & 14,4 & 8,7 & \\
\hline Parentes & 3,6 & 3,5 & 0,1118 & 1,9 & 1,8 & 0,6161 & 2,7 & 2,9 & 0,6305 & 8,1 & 4,8 & 0,1108 \\
\hline
\end{tabular}

Segue alguma dieta

\begin{tabular}{|c|c|c|c|c|c|c|c|c|c|c|c|}
\hline Sim & 12,7 & 7,9 & & 3,4 & 2,5 & & 3,8 & 3,1 & & 20,0 & 10,4 \\
\hline Não & 6,7 & 5,9 & $<0.0001$ & 2,6 & 2,3 & 0,0300 & 3,7 & 3,1 & 0,7779 & 13,0 & 8,3 \\
\hline
\end{tabular}

Preocupação quanto à caloria

\begin{tabular}{|c|c|c|c|c|c|c|c|c|c|c|c|}
\hline Sim & 10,7 & 7,1 & & 3,3 & 2,5 & & 3,5 & 2,8 & & 17,6 & 9,9 \\
\hline Não & 4,4 & 4,5 & $<0.0001$ & 2,1 & 2,1 & 0,0010 & 3,9 & 3,5 & 0,8373 & 10,3 & $<0.0001$ \\
\hline
\end{tabular}

Medo de engordar

$\begin{array}{lrrrrrrrrrrrrrrrr}\text { Sim } & 9,9 & 7,0 & & 3,2 & 2,4 & & 3,2 & 2,6 & 16,3 & 9,7 & & & & & & \\ \text { Não } & 3,1 & 3,0 & <\mathbf{0 . 0 0 0 1} & 1,7 & 1,9 & \mathbf{0 , 0 0 0 1} & 5,0 & 3,9 & \mathbf{0 , 0 1 4 9} & 9,7 & 5,3 & <\mathbf{0 . 0 0 0 1}\end{array}$

Frequenta bares e festas

\begin{tabular}{|c|c|c|c|c|c|c|c|c|c|c|c|c|}
\hline Frequentemente & 7,5 & 6,5 & & 2,9 & 2,2 & & 3,0 & 2,4 & & 13,4 & 8,3 & \\
\hline Às vezes & 8,3 & 7,1 & & 2,8 & 2,4 & & 3,7 & 3,2 & & 14,8 & 9,9 & \\
\hline Raramente & 8,4 & 7,2 & 0,8874 & 2,7 & 2,8 & 0,7522 & 4,6 & 3,5 & 0,0685 & 15,7 & 9,2 & 0,4248 \\
\hline
\end{tabular}

Realiza atividade física

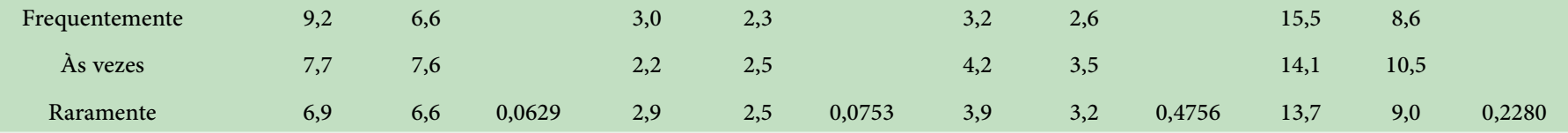

Tem doença crônica

\begin{tabular}{|c|c|c|c|c|c|c|c|c|c|c|c|c|}
\hline Sim & 9,9 & 6,9 & & 3,3 & 2,7 & & 3,6 & 2,9 & & 16,7 & 9,3 & \\
\hline Não & 7,8 & 6,8 & 0,1622 & 2,7 & 2,4 & 0,4420 & 3,7 & 3,1 & 0,8148 & 14,2 & 9,2 & 0,2031 \\
\hline \multicolumn{13}{|l|}{ Considera-se ansioso } \\
\hline Sim & 9,0 & 7,5 & & 3,1 & 2,6 & & 3,8 & 3,2 & & 15,9 & 10,1 & \\
\hline Não & 6,3 & 5,1 & 0,0356 & 2,1 & 1,7 & 0,0266 & 3,5 & 2,8 & 0,8278 & 11,9 & 6,6 & 0,0310 \\
\hline \multicolumn{13}{|l|}{ Considera-se triste } \\
\hline Sim & 10,7 & 7,3 & & 3,1 & 2,3 & & 4,5 & 2,9 & & 18,3 & 9,2 & \\
\hline Não & 7,8 & 6,8 & 0,0650 & 2,8 & 2,4 & 0,4303 & 3,6 & 3,1 & 0,0938 & 14,1 & 9,1 & 0,0290 \\
\hline \multicolumn{13}{|l|}{ Satisfação com o corpo } \\
\hline Satisfeito & 6,1 & 5,8 & & 2,3 & 2,1 & & 4,2 & 3,6 & & 12,7 & 8,3 & \\
\hline Pouco satisfeito & 9,2 & 7,1 & & 2,9 & 2,4 & & 3,1 & 2,3 & & 15,2 & 9,5 & \\
\hline Insatisfeito & 14,1 & 7,1 & $<0.0001$ & 5,3 & 2,1 & $<0.0001$ & 2,8 & 2,1 & 0,1814 & 22,3 & 8,6 & 0,0005 \\
\hline
\end{tabular}




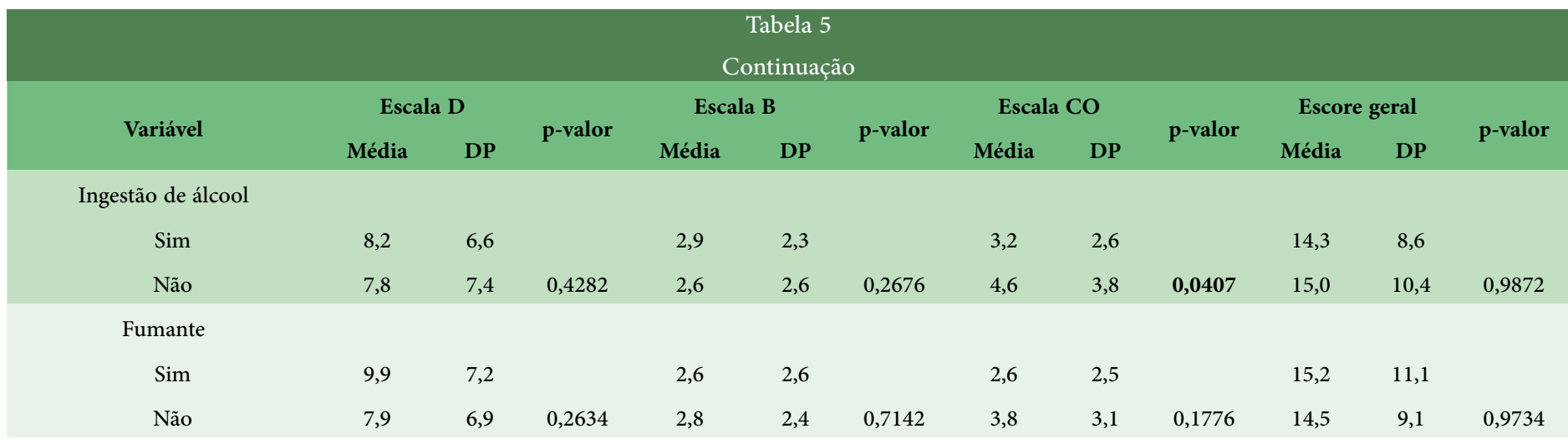

Fonte: Elaborada pelos autores.

\section{DISCUSSÃO}

Este estudo evidenciou que, no curso de Medicina investigado, havia um predomínio de estudantes do sexo feminino com riscos significativos de transtornos alimentares em relação aos homens. O fenômeno da feminização nas universidades iniciou-se a partir da década de 1920 com a implantação de políticas educacionais no período Vargas. Na medicina, especificamente, esse fenômeno ocorreu a partir dos anos 1970. Entre as décadas de 1970 e 1990, houve na Universidade Federal do Rio de Janeiro (UFRJ) um acréscimo de $27 \%$ do contingente feminino no curso de graduação em Medicina ${ }^{11}$.

Atualmente, na América Latina vem ocorrendo uma crescente feminização da profissão médica ${ }^{12}$. Esse fenômeno ocorre principalmente no grupo de 29 anos ou menos. Esses achados corroboram os resultados deste estudo que evidenciou que, dentre 162 estudantes internos do curso de Medicina pesquisado, 90 eram do sexo feminino e a maioria com até 24 anos de idade ${ }^{12}$.

O maior escore na escala EAT-26 no sexo feminino corrobora os achados de uma pesquisa que identificou que as mulheres apresentaram $17 \%$ a mais de chance de desenvolver transtornos alimentares ${ }^{5}$. Um outro estudo encontrou uma taxa significativamente maior entre as mulheres (40\%) do que entre os homens $(28,1 \%)$ da Universidade de Notre Dame, no Estados Unidos, para o desenvolvimento de transtornos alimentares.

Essa realidade é justificada pela maior influência que a mídia e a sociedade exercem sobre as mulheres no que diz respeito a um padrão estético corporal considerado ideal, em que a magreza é vista como saúde ${ }^{13}$. Para alcançarem esse padrão, muitas vezes práticas não saudáveis são adotadas, desencadeando, por exemplo, bulimia nervosa e anorexia, o que explica os maiores escores do sexo feminino nas escalas D e B do EAT-26 ${ }^{13}$.

Um maior escore na escala $D$ foi encontrado em estudantes que afirmaram que trabalhavam e estudavam. Tal fato se deve à escassez de tempo do estudante de Medicina ocasionada por uma rotina tumultuada, dificultando o preparo de alimentos e impossibilitando que as refeições sejam feitas em horários adequados. Em geral, os hábitos alimentares dos estudantes universitários são caracterizados por alta ingesta de alimentos, como salgadinhos, fast-foods, batata frita, tortas, bolos, refrigerantes e uma baixa ingesta de frutas e vegetais. $\mathrm{O}$ tempo gasto na universidade é considerado, desse modo, um empecilho para a prática de uma alimentação saudável ${ }^{14}$.

Foi identificado no estudo que os estudantes com sobrepeso apresentaram maior escore na escala D. Esse fato demonstra uma preocupação exagerada com a comida e com a aparência física. A fim de se enquadrarem no padrão de beleza instituído atualmente, terem maior aceitação no meio social e obterem emagrecimento rápido, as pessoas adotam comportamentos de riscos diretamente ligados aos transtornos alimentares ${ }^{15-17}$. Contudo, maior escore na escala $\mathrm{CO}$ foi encontrado em indivíduos abaixo do peso, o que demonstra um autocontrole sobre a própria alimentação ${ }^{15-17}$.

Os estudantes que relataram seguir alguma dieta, os que afirmaram ter preocupação com a quantidade de calorias ingeridas e aqueles que têm medo de engordar obtiveram maiores escores nas escalas D e B e no escore geral da escala EAT-26. Essas três dimensões do fenômeno estão correlacionadas, já que refletem um comportamento em que a gordura deve ser evitada. Sentir medo ou pavor da ideia de estar engordando é algo frequente em pessoas com transtornos alimentares, o que leva a uma maior preocupação com a quantidade de calorias dos alimentos consumidos; nesse caso, as pessoas optam por alimentos com poucas calorias, tidos como mais saudáveis ${ }^{15}$.

$\mathrm{Na}$ verdade, esses futuros médicos sentem-se, na maioria das vezes, obrigados a seguir os rigorosos padrões estéticos existentes relacionados com a manutenção da boa forma por causa da falsa ideia de que, para realizar a promoção da saúde, devem apresentar-se saudáveis, o que, na sociedade atual, é sinônimo de magreza. Portanto, os acadêmicos estão inseridos em um ambiente favorável ao desenvolvimento de transtornos alimentares na graduação ${ }^{18}$.

$\mathrm{Na}$ anorexia nervosa, por exemplo, há uma supervalorização da forma corporal. Por conta disso, o indivíduo recusa alimentos calóricos porque tem um medo intenso de ganhar peso e o desejo persistente de emagrecer. Já na bulimia nervosa, por medo de engordar, os indivíduos induzem o próprio vômito e/ou fazem o uso de laxantes, logo após a ingesta de alimentos hipercalóricos, para a prevenção do ganho de peso ${ }^{19}$. A preocupação constante com a ingesta de calorias, a adoção de dietas da moda sem prescrição de nutricionista e o contínuo medo de engordar podem ser indícios do desenvolvimento de transtornos maiores, como a anorexia nervosa e a bulimia nervosa.

Essa convergência de fatores pode levar a comportamentos de risco que interferem nos hábitos alimentares, como restrição severa por dietas rígidas e altos níveis de atividades físicas ${ }^{20}$. Ademais, a insatisfação corporal direcionada à magreza influencia sobremaneira no desenvolvimento da

\begin{tabular}{l|l}
7 & REVISTA BRASILEIRA DE EDUCAÇÃ̃ MÉDICA \\
\hline $44(3)$ : e097; 2020
\end{tabular} 
restrição alimentar e de sintomas relacionados à anorexia e à bulimia ${ }^{21}$.

O desenvolvimento da restrição alimentar, por sua vez, ocorre por estratégias desenvolvidas pelo próprio indivíduo para reduzir e eventualmente extinguir o apetite. Uma das estratégias consiste em pensar imediatamente nas consequências negativas que a ingesta de alimentos altamente calóricos pode provocar na saúde, no peso e na aparência corporal. Outra estratégia bastante eficaz e tida como o protótipo da anorexia nervosa é a exposição prolongada e frequente aos alimentos, porém sem realizar a ingesta deles ${ }^{20}$.

Os estudantes que participaram da pesquisa e que se consideraram ansiosos, tristes e insatisfeitos com o próprio corpo apresentaram maiores escores na escala EAT-26, demonstrando maior probabilidade de desenvolver transtornos alimentares. Esse achado corrobora uma pesquisa que evidenciou níveis anormais de hormônios responsáveis pela regularização do sono, apetite, humor e estresse em alunos com distúrbios alimentares ${ }^{16}$. Muitos indivíduos recorrem à ingesta copiosa como ferramenta para aliviar a ansiedade e problemas encontrados no dia a dia e, ao perceberem o ganho de peso advindo dessa prática, sentem-se tristes e insatisfeitos com o próprio corpo ${ }^{6,22,23}$.

Estudos com a utilização do EAT-26 encontraram relação significativa entre a insatisfação corporal e comportamentos alimentares de risco, sendo considerado um dos principais aspectos para diagnosticar os transtornos, já que pode resultar em prejuízos no comportamento (baixa autoestima, comparação social, aumento de procedimentos estéticos, ansiedade, diminuição da qualidade de vida e ideação suicida) e atitudes alimentares com maior propensão a desenvolver distúrbios ${ }^{24,25}$.

Dessa forma, os transtornos alimentares, em especial a anorexia nervosa e a bulimia nervosa, são síndromes psiquiátricas complexas que causam distorções cognitivas relacionadas à alimentação e ao peso corporal, frequentemente relacionadas a outros distúrbios psíquicos (transtorno depressivo, abuso de substâncias e transtorno de ansiedade, por exemplo), com um profundo impacto social e físico ${ }^{26}$.

Os indivíduos tendem a estabelecer um comportamento de isolamento social (abandonando atividades sociais, físicas, ocupacionais ou de lazer) tanto pela perda da confiança - estão insatisfeitos com o próprio corpo e têm medo de sofrer discriminação no ambiente em que se encontram, no caso daqueles que estão acima do peso considerado ideal - quanto pela necessidade compulsiva de manter uma dieta altamente restritiva e/ou a prática de exercícios físicos ${ }^{27}$. Essas práticas aumentam os riscos de surgimento de uma desordem psíquica (depressão, fobias, atitudes compulsivas e repetitivas) e, consequentemente, a perpetuação de transtornos alimentares por meio de um círculo vicioso.

\section{CONCLUSÃO}

Diante dos resultados deste trabalho, identificou-se que, da amostra dos 162 estudantes internos de Medicina analisados, um número significativo obteve pontuação que os classifica com indicador positivo para uma possível existência de anorexia nervosa e bulimia nervosa.

Ficaram evidenciados maiores escores na escala EAT-26 em estudantes internos de Medicina que são do sexo feminino, que trabalham e estudam e naqueles com sobrepeso. Além disso, seguir alguma dieta, possuir preocupação quanto à quantidade de calorias, ter medo de engordar, ser ansioso, triste e insatisfeito com próprio corpo também foram fatores associados com maiores escores. Todos esses fatores podem ser relacionados com um maior risco de esses estudantes desenvolverem distúrbios alimentares, como a anorexia e a bulimia.

Os resultados encontrados nesta pesquisa podem auxiliar sobremaneira na identificação das características dos estudantes de Medicina que possuem risco de desenvolver qualquer transtorno alimentar. Com base nos fatores associados evidenciados neste estudo, os gestores poderão adotar ações de prevenção e intervenção com foco direcionado às características de estudantes que possam desenvolver transtornos alimentares.

Portanto, os achados se constituem em uma ferramenta para que as instituições acadêmicas cheguem até esse grupo de risco e desenvolvam programas específicos de atendimento a esses estudantes, de forma que promovam tratamento precoce e ofereçam todo o suporte necessário, com o propósito de diminuir a incidência de sérias consequências envolvendo esses indivíduos. Além disso, programas de prevenção podem ser desenvolvidos nesse ambiente estudantil, já que, como visto, a graduação é um ambiente propício para o aparecimento desses transtornos.

\section{REFERÊNCIAS}

1. Ciao AC, Ohls OC, Pringle KD. Should body image programs be inclusive? A focus group study of college students. Int J Eat Disord. 2017;51(1):82-6.

2. Nunes LG, Santos MCS, Souza AA. Fatores de risco associados ao desenvolvimento de bulimia e anorexia nervosa em estudantes universitários: uma revisão integrativa. HU Rev. 2017;43(1):61-9.

3. Torres CP, Espinoza KT, Pérez CS, Viteri-Condori L, Aguilar JC, Rondan AT. Trastornos de la conducta alimentaria en estudiantes de medicina de una Universidad de Perú. Rev Cuba Salud Pública. 2017;43(4):552-63.

4. Chammas R, Bou Mosleh J, Jaalouk D, Bou Mitri C, Aoun A. Eating disorders among university students in a middle eastern urban setting: who is at risk? Clin Nutr. 2017;36(1):S113-S114.

5. Lipson SK, Sonneville KR. Eating disorder symptoms among undergraduate and graduate students at 12 U.S. colleges and universities. Eat Behav. 2017;24:81-8.

6. Tavolacci MP, Grigioni S, Richard L, Meyrignac G, Déchelotte P, Ladner J. Eating disorders and associated health risks among university students. J Nutr Educ Behav. 2015;47(5):412-20.

7. Costa MFL, Barreto SM. Tipos de estudos epidemiológicos: conceitos básicos e aplicações na área do envelhecimento. Epidemiol Serv Saúde. 2003;12(4):189-201.

8. Garner DM, Garfinkel PE. The Eating Attitudes Test: an index of the symptoms of anorexia nervosa. Psychol Med. 1979;9(2):273-9.

9. Nunes MA, Bagatini LF, Abuchaim AL, Kunz A, Ramos D, Silva JA, et al. Distúrbios da conduta alimentar: considerações sobre o Teste de Atitudes Alimentares (EAT). Rev ABP-APAL. 1994;16(1):7-10.

10. Garner DM, Olmsted MP, Bohr Y, Garfinkel PE. The eating attitudes test: psychometric features and clinical correlates. Psychol Med. 1982;12(4):871-8.

11. Minella LS. Medicina e feminização em universidades brasileiras: o gênero nas interseções. Rev Estud Fem. 2017;25(3):1111-28.

12. Petrone P. La feminizacíon en la Medicina. Rev Colomb Cir. 2018;33(2):132-4.

13. Gupta N, Bhargava R, Chavan BS, Sharan P. Eating attitudes and

8 REVISTA BRASILEIRA DE EDUCAÇ̄̃o MÉDICA

8 44 (3) : e097; 2020 
body shape concerns among medical students in Chandigarh. Indian J Soc Psychiatry. 2017;33(3):219-24.

14. Bernardo GL, Jomori MM, Fernandes AC, Proença RPC. Food intake of university students. Rev Nutri. 2017;30(6):847-65.

15. Oliveira SD, Caires TF, Silva EF, Silva CR, Viana SDL. Percepção da imagem corporal e atitudes alimentares de modelos em São Paulo. RBONE. 2010;12(76):1157-63.

16. Panchami, Samuel T. A cross-sectional study of disturbed eating attitudes and behaviours in medical students. Int. J Res Med Sci. 2016;4(7):2830-3.

17. Vargas LM, Cantorani JRH, Mauricio LK, Annunciato SKL. Nível de atividade física de estudantes universitários com e sem distúrbio da imagem corporal. Pensar Prát. 2016;19(1):169-81.

18. Miranda AS, Caíres SN, Castro JS. Transtornos alimentares e imagem corporal em universitárias. Rev Integrart. 2017;1(1):62-73.

19. Souza S, Verrengia EC. Autopercepção da imagem corporal e prevalência de comportamentos sugestivos de anorexia nervosa em universitários. Rev Uningá. 2012;34(1):23-31.

20. Jansen A. Eating disorders need more experimental psychopathology. Behav Res Ther. 2016;86:2-10.

21. Fortes LS, Ferreira MEC, Oliveira SFM, Paes PP, Almeida SS. Influência da insatisfação corporal direcionada à magreza na restrição alimentar e nos sintomas bulímicos: uma investigação prospectiva com jovens nadadoras. Rev Bras Ciênc Esporte. 2018;40(3):242-7.

22. Bermúdez P, Machado K, García I. Transtorno del comportamento alimentario de difícil tratamento. Caso clínico. Arch Pediatr Urug. 2016;87(3):240-4.

23. Pitanupong J, Jatchavala C. Atypical eating attitudes and behaviors in Thai Medical Students. Siriraj Med J. 2017;69(1):5-10.

24. Fortes LS, Amaral ACS, Almeida SS, Conti MA, Ferreira MEC. Qualidades psicométricas do Eating Attitudes Test (EAT-26) para adolescentes brasileiros do sexo masculino. Psic Teor Pesq. 2016;32(3):1-7.

25. Souza AC, Alvarenga MS. Insatisfação com a imagem corporal em estudantes universitários - uma revisão integrativa. J Bras Psiquiatr. 2016;65(3):286-99.

26. Ramaiah RR. Eating disorders among medical students of a rural teaching hospital: a cross-sectional study. Int J Community Med Public Health. 2015;2(1):25-8.

27. Oliveira ROA, Veras KMA. Prevalência de vigorexia e ortorexia em estudantes de graduação. Rev Científica UMC. 2018;3(3):1-4.

\section{CONTRIBUIÇÃO DOS AUTORES}

Mariana de Oliveira Inocente Aidar, Rafaela Borges de Freitas e Rogério José de Almeida participaram da concepção e do planejamento do projeto de pesquisa, da coleta de dados, da análise e interpretação dos dados, da redação e da revisão final.

Gabriela Cunha Fialho Cantarelli Bastos, Aline Alves Brasileiro e Antonio Márcio Teodoro Cordeiro Silva participaram da análise e interpretação dos dados, da redação e da revisão final.

\section{CONFLITO DE INTERESSES}

Não há.

\section{ENDEREÇO PARA CORRESPONDÊNCIA}

Rogério José de Almeida. Avenida Universitária, 1440, área 4, bloco K, Departamento de Medicina, Setor Universitário, Goiânia, GO, Brasil. CEP: 74605-010.

E-mail: rogerioufg@hotmail.com 